Revue d'histoire de l'Amérique française

REYUE D.HISTOIRE DE L'AMÉRIQUE FRANÇAISE

\title{
Le bétail et l'activité économique en Nouvelle-France
}

La vente et la location

\section{Valérie D’Amour et Évelyne Cossette}

Volume 56, numéro 2, automne 2002

URI : https://id.erudit.org/iderudit/007317ar

DOI : https://doi.org/10.7202/007317ar

Aller au sommaire du numéro

Éditeur(s)

Institut d'histoire de l'Amérique française

ISSN

0035-2357 (imprimé)

1492-1383 (numérique)

Découvrir la revue

Citer cette note

D’Amour, V. \& Cossette, É. (2002). Le bétail et l'activité économique en Nouvelle-France : la vente et la location. Revue d'histoire de l'Amérique française, 56(2), 217-233. https://doi.org/10.7202/007317ar
Résumé de l'article

Les historiens du Québec préindustriel ont souvent traité d'agriculture, mais ils ont peu abordé la question de l'élevage, du moins en tant qu'activité spécifique. Ainsi, en analysant les contrats de vente et de location d'animaux passés à Montréal entre 1661 et 1761, nous nous interrogeons sur le rôle que pouvait jouer le bétail dans les relations économiques des résidants de l'île selon leur appartenance à un groupe professionnel. Nous observons d'abord, qu'il soit question de vente ou de location, une diversité étonnante des profils professionnels des preneurs et davantage des propriétaires. Par contre, le bétail devient un moyen d'échange particulièrement important pour l'élite urbaine et pour les paysans pour qui les animaux sont un avoir indispensable. 


\section{NOTE DE RECHERCHE}

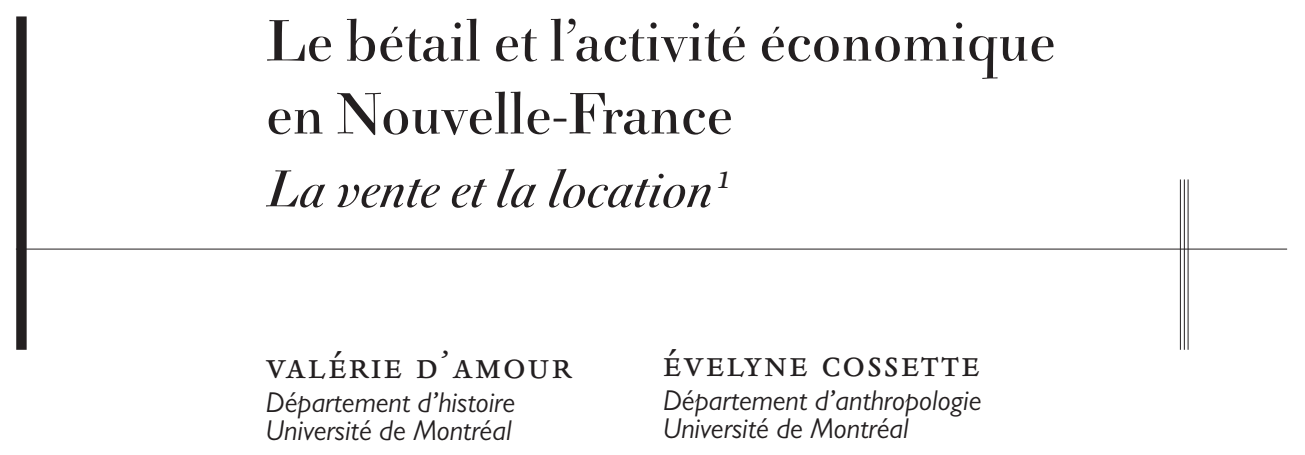

RÉSUMÉ - Les historiens du Québec préindustriel ont souvent traité d'agriculture, mais ils ont peu abordé la question de l'élevage, du moins en tant qu'activité spécifique. Ainsi, en analysant les contrats de vente et de location d'animaux passés à Montréal entre |66 | et $|76|$, nous nous interrogeons sur le rôle que pouvait jouer le bétail dans les relations économiques des résidants de l'île selon leur appartenance à un groupe professionnel. Nous observons d'abord, qu'il soit question de vente ou de location, une diversité étonnante des profils professionnels des preneurs et davantage des propriétaires. Par contre, le bétail devient un moyen d'échange particulièrement important pour l'élite urbaine et pour les paysans pour qui les animaux sont un avoir indispensable.

ABSTRACT - Although historians have frequently studied agricultural developments in pre-industrial Quebec, they have rarely considered livestock raising as a specific activity. Through an analysis of deeds of sale and leases of animals on the island of Montréal between I66I and I76I, we examine the role of cattle and horses in the economic relations of the island's inhabitants. Regardless of the type of transfer, the occupational profile of buyers and lessees shows great diversity, even more so for the owners. Nonetheless, it is clear that livestock was becoming an important medium of exchange for the urban élite, and for farmers for whom cattle represented an indispensable asset.

1. Cette recherche a reçu l'appui du fonds du CRSH. Nous remercions sincèrement Thomas Wien et Laurence Johnson de leurs critiques et commentaires judicieux. 
I AGRICUlTURE OCCUPE une place prépondérante dans l'historiographie années, son histoire aux $\mathrm{XVII}^{\mathrm{e}}$ et $\mathrm{XVIII}^{\mathrm{e}}$ siècles s'est considérablement renouvelée. Traditionnellement, les historiens partaient de l’idée que la terre était facilement disponible en Amérique et dépeignaient une société rurale où l’inégalité sociale demeurait beaucoup moins forte qu'en Europe à la même époque; ils croyaient que chaque ménage en milieu rural possédait ou allait tôt ou tard posséder ses terres et le matériel nécessaire à leur exploitation ${ }^{2}$. Grâce à l'étude de sujets tels que les outils et les techniques agricoles, l'occupation des sols, les besoins en capitaux et les variations de la production, les historiens ont démontré que cette conception relevait plutôt du mythe, la réalité vécue étant en définitive beaucoup plus complexe. En effet, si la terre était accessible en contexte de colonisation, son exploitation demandait un capital de départ, des méthodes et des aptitudes qui font que certains colons réussissaient mieux que d'autres à se tirer d'affaire ${ }^{3}$. Pour paraphraser une formule de Sylvie Dépatie, l'égalité dans l'accès à la terre n'impliquait pas pour autant l'uniformité de la paysannerie, dont les membres devaient composer avec des terres de qualité variable et des moyens de départ différents selon les conditions sociales des parents notamment ${ }^{4}$.

Dans leurs travaux, les historiens n'ont pas manqué de souligner le rôle indispensable du bétail dans l'exploitation agricole en tant que capital, force de traction et élément de l'alimentation familiale. Certains font même du bétail un élément de différenciation sociale. C’est le cas de Christian Dessureault, qui a démontré que la possession de bétail et d'outils agricoles, plus particulièrement d'une charrue et d'un train de labour, n'est pas l'apanage de tous les cultivateurs de Saint-Hyacinthe, même au début du XIX ${ }^{\mathrm{e}}$ siècle $^{5}$.

2. Par exemple, Guy Frégault, La Civilisation de la Nouvelle-France (1713-1744) (Montréal, Société des éditions Pascal, 1944), 285 p.

3. Louise Dechêne montre, notamment au chapitre IV, «L'occupation du sol», dans Habitants et marchands de Montréal au XVII ${ }^{e}$ siècle (Montréal, Boréal Express 1988), 532 p., que le défrichement d'une terre est un travail long et ardu qui demande un bon capital de départ. Le manque d'argent ou d'intérêt dans la culture de la terre, tout comme une situation familiale précaire provoquée par exemple par la mort de l'épouse, peut encourager le colon à travailler comme journalier ou à louer une terre, ce qui retarde la mise en culture de ses propres terres et se répercute éventuellement dans l'établissement des générations futures.

4. Sylvie Dépatie. "La structure agraire au Canada : le cas de l'île Jésus au xviII ${ }^{\mathrm{e}}$ siècle", Communications historiques/Historical Papers (1986) : 56-85.

5. Christian Dessureault, «L'égalitarisme paysan dans l'ancienne société rurale de la vallée du Saint-Laurent : éléments pour une réinterprétation ", Revue d'histoire de l'Amérique française, 40,3 (hiver 1987) : 373-407. 
Cependant, peu de chercheurs ont tenté de percevoir l'élevage comme une activité spécifique. Ils le considèrent plutôt comme une composante certes indispensable de la polyculture, mais finalement d'un intérêt secondaire, la production de céréales demeurant, au Canada préindustriel, l'activité principale d'intégration de la famille paysanne dans l'économie de marché 6 . Nous pensons qu'il ne faut pas sous-estimer l'importance de l'élevage dans cette société. Nous nous appuyons sur l'ouvrage de l'historien français Jean-Marc Moriceau, intitulé L'élevage sous l'Ancien Régime (XVI ${ }^{e}$ $X V I I I^{e}$ siècles) $)^{7}$, comme point de départ de notre réflexion. L'auteur y fait une synthèse de la situation de l'élevage en France du $\mathrm{xvI}^{\mathrm{e}}$ au $\mathrm{xvIII}{ }^{\mathrm{e}}$ siècle, tout en soulignant les particularités spécifiques à chaque région du royaume. Son étude l'amène à constater que la possession du bétail est une préoccupation pour tous les membres de la société rurale d'Ancien Régime, d'abord en raison de son utilité dans l'exploitation agricole et de son apport alimentaire, mais aussi à cause de son rôle d'instrument de distinction sociale. Le nombre de bêtes possédées, leur espèce et leur qualité déterminent une hiérarchie sociale complexe. Dans la compréhension de cet ordre social, l'auteur met aussi en lumière ce qui est longtemps demeuré selon lui «la face cachée de la propriété du bétail» : la location du cheptel. Placement souvent plus rentable que la rente foncière pour placer une épargne ou pour réinvestir des capitaux, la location de bétail permet aux paysans moins bien nantis d'obtenir pour un moment un apport supplémentaire ou le cheptel nécessaire à l'exécution de leurs travaux agricoles. Les observations de Moriceau le portent à conclure que l'élevage dans la France d'Ancien Régime ne représente pas seulement un appendice de l'agriculture :

loin d'être une occupation par défaut, il [l'élevage] constitua souvent l'activité la plus lucrative des économies régionales; loin d'être un simple môle de stabilité, il fut le théâtre d'une intense mobilité structurelle avec d'indéniables réorientations; loin d'être enfin un simple appoint alimentaire, il représenta,

6. Des historiens comme Guy Frégault ont souligné l’importance de la production de céréales dans l'économie de la colonie : «La terre est donc le principal arc-boutant de l'économie canadienne. Du moment que ses champs produisent moins, le pays s'anémie dangereusement», dans op. cit., 68. Plusieurs autres historiens ont abordé la question de l'agriculture dans le Québec préindustriel et la production de céréales, par exemple Maurice Séguin, La nation canadienne et l'agriculture (1760-1850) (Trois-Rivières, Boréal Express, 1970), ou encore Thomas Wien, «Les travaux pressants : calendrier agricole, assolement et productivité au Canada au $\mathrm{xvIII}^{\mathrm{e}}$ siècle», Revue d'histoire de l'Amérique française, 43,4 (printemps 1990) : 535-558.

7. Jean-Marc Moriceau, L'élevage sous l'Ancien Régime (XVI ${ }^{e}$-XVIII ${ }^{e}$ siècles) (Paris, Sedes, 1999), $256 \mathrm{p}$. 
presque toujours, l'instrument privilégié de l'insertion paysanne dans les échanges ${ }^{8}$.

Dans ce sens, deux types d'élevage sont présents dans le paysage français d'alors : un élevage de subsistance, omniprésent, et un élevage spéculatif concentré dans des régions plus spécifiques, comme les pays de grands pâturages, et pratiqué par des groupes sociaux tels que les marchands des villes. Donc, en plus de constituer un avoir indispensable, le bétail en France fait aussi l'objet de transactions avantageuses, il joue un rôle non négligeable dans les relations économiques de la paysannerie et les échanges entre les mondes rural et urbain.

Qu'en est-il de la place du bétail comme moyen d'échange dans la société canadienne à l'époque de la Nouvelle-France? Il est possible de trouver dans l'historiographie des traces de l'activité économique impliquant le bétail. Ainsi, Robert-Lionel Séguin met en lumière la pratique de la location du bétail à Montréal, pratique qu'il décrit cependant davantage qu'il ne l'analyse ${ }^{9}$. Louise Dechêne souligne aussi, brièvement, la distribution inégale des bêtes et le recours à la location pour combler les besoins des défricheurs en bétail ${ }^{10}$. De plus, des études ont montré l'importance des relations économiques entre la ville et la campagne dans la région montréalaise, relations auxquelles participe le bétail. Pensons à l'analyse de Jocelyne Perrier sur les tanneurs de la région montréalaise, qui entretiennent des liens d'affaires importants avec les bouchers de la ville pour les approvisionner en peaux d'animaux, et à celle de Jennifer L. Waywell qui montre, par l'étude de baux à ferme, les interactions entre la possession de terres et celle de bétail par des investisseurs urbains et leur exploitation par des ouvriers agricoles ${ }^{11}$.

À la suite de ces travaux, par l'examen spécifique des contrats de vente et de location de bovins et de chevaux passés sur l'île de Montréal entre 1661 et 1761, nous voulons comprendre un peu mieux le rôle du bétail dans les relations économiques que tissent entre eux les habitants de l'île, qu'ils soient citadins ou ruraux. Dans un premier temps, nous identifierons

8. Ibid., 205.

9. Robert-Lionel Séguin, La civilisation traditionnelle de l'habitant aux XVII ${ }^{e}$-XVIII ${ }^{e}$ siècles (Montréal, Fides, 1967), 228-240.

10. Louise Dechêne, "La gestion de l'habitation", dans op. cit., 314-320.

11. Jocelyne Perrier, Tanneurs et tanneries dans le gouvernement de Montréal au XVIII ${ }^{e}$ siècle, mémoire de maîtrise (histoire), Université de Montréal, 2002, 136 p. ; Jennifer L. Waywell, Farm Leases and Agriculture on the Island of Montreal, 1780-1820, mémoire de maîtrise (histoire), Université McGill, 1989, 178 p. 
les groupes socioprofessionnels qui demeurent les plus engagés dans ces transactions, que ce soit comme bailleurs/vendeurs ou comme locataires/acheteurs. Nous tenterons de voir par la suite s'il n'existe qu'un modèle-type ou s'il se présente une diversité de situations qui explique le commerce des animaux. Mais attardons-nous pour commencer à l'analyse de la source proprement dite.

\section{LA SOURCE}

\section{Le corpus et sa représentativité}

Le repérage des transactions concernant le bétail a pu être aisément effectué à l'aide de Parchemin, inventaire des actes notariés passés avant $1775^{12}$. Pour les fins de cette analyse, seuls les actes impliquant au moins un résidant de l'île de Montréal ont été retenus. Certains types de transactions ont été exclus de notre analyse, soit les cessions, les dons et les échanges, parce que leur faiblesse numérique - ils ne représentent que $3 \%$ de l'ensemble des contrats recensés - ne nous permettait pas d'établir des barèmes de comparaison. Il en va de même pour les contrats faisant référence aux brebis, génisses, poulains et pouliches, regroupant eux aussi seulement $3 \%$ des actes ${ }^{13}$. Soulignons enfin que les actes retenus peuvent porter sur une ou plusieurs têtes de bétail. Au total, notre corpus comprend 213 documents notariés concernant des bœufs, des chevaux, des vaches et des taures, soit 132 baux et 81 contrats de vente (tableau 1). Il faut cependant

TABLEAU 1

Nombre de contrats de vente et de location selon les types d'animaux

\begin{tabular}{|c|c|c|c|}
\hline & Bail & Vente & Total \\
\hline Bœufs et taureaux & 26 & 28 & 54 \\
\hline Vaches et taures & 98 & 7 & 105 \\
\hline Chevaux et juments & 3 & 30 & 33 \\
\hline Bestiaux & 5 & 16 & 21 \\
\hline Total & 132 & 81 & 213 \\
\hline
\end{tabular}

12. Hélène Lafortune et Normand Robert, Parchemin : banque de données notariales, 1635-1765 [cédérom] (Montréal, Société de recherche archiv-histo/Chambre des notaires du Québec/Archives nationales du Québec, 1996).

13. Louise Dechêne, op. cit. 
dire que la fréquence relative des ventes et locations, du moins celles qui font l'objet d'un contrat, varie énormément selon les types d'animaux. Les vaches, qui représentent près de la moitié du corpus, sont généralement louées, alors que les chevaux sont vendus. Les bœufs sont presque autant loués que vendus.

Un total de 213 contrats sur une période de 100 ans donne en moyenne deux contrats par année. Est-ce à dire que la location et la vente de bétail sont en réalité des phénomènes rares à Montréal à l'époque de la NouvelleFrance? Il est plus probable qu'en raison, entre autres, du coût des services d'un notaire, bon nombre de transactions ne faisaient tout simplement pas l'objet d'un contrat notarié. Les prêts ou ventes d'animaux entre deux voisins par exemple. Par ailleurs, l'analyse de la distribution des contrats par décennie (tableau 2) montre que le nombre d'actes varie considérablement dans le temps. En effet, 185 contrats, soit $86 \%$ de l'ensemble, sont signés entre 1660 et 1710, et parmi eux 111 (52\%) entre 1691 et 1710. Quelles peuvent être les raisons d'une telle concentration? À la fin du $\mathrm{xvII}^{\mathrm{e}}$ siècle, la population de Montréal augmente, passant de 659 résidants en 1666 à 1388 en 1681 et à environ 3605 en $1706^{14}$. La demande en bétail croît parallèlement, ce qui pourrait expliquer l'augmentation du nombre d'actes contractés. Le colon s'établissant sur une terre ne possède pas toujours les capitaux nécessaires à l'acquisition d'animaux, comme nous l'avons vu précédemment; c'est ce qui explique le nombre considérable de baux au début de la colonisation. Le revirement est sensible dès la première décennie du $\mathrm{xVIII}^{\mathrm{e}}$ siècle, alors que le nombre de ventes supplante pour une première fois celui des locations.

Enfin, une chute brutale du nombre de contrats s'observe à partir de 1710. Les bêtes étant disponibles en plus grande quantité et leur valeur étant à la baisse ${ }^{15}$, peut-être faisaient-elles plus rarement l'objet de contrats notariés vu le coût des services du notaire. De même, l'augmentation de la population s'effectuant alors davantage par l'accroissement naturel que par l'immigration ${ }^{16}$, il est possible que les gens se côtoyant et se connaissant bien aient tendance à conclure des ententes verbales et à diminuer leur

14. Ibid., 492.

15. À titre d'exemple, nous avons remarqué dans les contrats analysés qu'entre 1691 et 1700 , la proportion la plus importante de vaches (37\%) vaut entre 40 et 59 livres, alors qu'au cours de la décennie suivante la proportion prédominante (38\%) a une valeur variant entre 20 et 39 livres. La situation est semblable pour les bœufs.

16. Louise Dechêne, op. cit., 98 . En 1715, les immigrants représentent de $10 \%$ à $15 \%$ de la population. 
TABLEAU 2

Les contrats de vente et de location recensés selon les décennies

\begin{tabular}{c|c|c|c}
\hline & Location & Vente & Total \\
\hline $1661-1670$ & 7 & 3 & 10 \\
$1671-1680$ & 22 & 8 & 30 \\
$1681-1690$ & 27 & 7 & 34 \\
$1691-1700$ & 40 & 17 & 57 \\
$1701-1710$ & 24 & 30 & 54 \\
$1711-1720$ & 3 & 8 & 11 \\
$1721-1730$ & 1 & 3 & 4 \\
$1731-1740$ & 0 & 2 & 2 \\
$1741-1750$ & 2 & 0 & 2 \\
$1751-1760$ & 132 & 3 & 9 \\
\hline
\end{tabular}

recours au notaire. Il est aussi fort probable que les parents participent à l'établissement de leurs enfants en leur fournissant des animaux provenant de leur cheptel, ce qui n’apparaît pas dans les actes notariés. Disons à cet effet que l'étude des contrats de mariage pourrait amener des précisions intéressantes.

Ainsi, quoique plusieurs transactions concernant le bétail demeurent informelles, sans contrat, l'analyse des actes recensés de vente et de location nous permettra tout de même de donner un aperçu des groupes professionnels auxquels appartiennent les parties intéressées dans ces transactions à la fin du XvII ${ }^{\mathrm{e}}$ et au début du XviII ${ }^{\mathrm{e}}$ siècle dans l'île de Montréal, comme de comprendre les mécanismes socio-économiques qui les sous-tendent.

\section{Les contrats types de location et de vente}

Quelle que soit l'espèce de l'animal, les contrats de location passés devant notaire contiennent essentiellement les mêmes informations. La présence et la précision de ces informations dépendent cependant de la vigilance et du souci du détail du notaire. L'acte commence toujours par l'identification des parties. Le bailleur est présenté en premier lieu. La profession du bailleur et celle du locataire sont inscrites une fois sur deux, alors que les lieux de résidence le sont dans $90 \%$ des cas. Le notaire indique la durée de la location, durée très variable selon les types d'animaux et pouvant aller de 
quelques semaines à plusieurs années (tableau 3). L’animal loué fait par la suite l'objet d'une description plus ou moins détaillée. Habituellement sont indiqués son âge, sa couleur et sa valeur. Encore une fois, la qualité des informations retrouvées varie ${ }^{17}$. Enfin, vient l'énumération des conditions de location. Celles-ci se divisent en trois parties distinctes. D’abord, le notaire mentionne les soins à apporter à l'animal. Le preneur sera tenu de bien nourrir, loger et soigner l'animal ou les animaux, été comme hiver. Le bailleur fixe dans un deuxième temps le loyer, qui devra être versé en nature, c'est-à-dire en services ou en biens de toutes sortes allant des journées de travail aux livres de beurre, ou en argent. Cette partie comporte aussi les informations relatives au sort de la progéniture, lorsqu'il s'agit d'une femelle. Puis, il est indiqué ce qui arrivera en cas de mort naturelle ou accidentelle de l'animal. Plusieurs ententes peuvent être envisagées. Pour une mort naturelle, les deux clauses les plus fréquentes sont le partage de la perte à parts égales entre le preneur et le bailleur et la perte assumée par le bailleur. Dans ce cas, le locataire doit remettre la peau de l'animal qui sera vendue par le bailleur pour éponger une partie de la perte. Advenant la mort de l'animal par la faute du locataire, généralement celui-ci doit en rembourser la valeur au propriétaire. Nous avons relevé quelques clauses particulières. Par exemple, deux actes spécifient qu’en cas de mort par une attaque des Iroquois, le preneur devra remettre, en partie ou en totalité, la valeur de la bête au bailleur ${ }^{18}$. Ou encore, lorsque Jacques Charbonnier loue une vache à Pierre Goguet pour deux ans, une clause stipule qu'en cas de départ pour la France du bailleur, la location sera annulée $e^{19}$.

Les contrats de vente contiennent grosso modo les mêmes informations : la désignation des parties, la description de l'animal ou des animaux vendus, les conditions de vente. Ces dernières se résument en général par le paiement en argent ou en matériel de la valeur de l'animal, souvent en plusieurs versements à date fixe.

17. Pour les vaches, nous connaissons les informations sur leur couleur dans $69 \%$ des cas, sur leur âge dans $68 \%$ des cas et sur leur valeur dans $72 \%$ des cas. Pour les bœufs, la couleur est mentionnée à $63 \%$, l'âge à $78 \%$ et la valeur à seulement $59 \%$. Nous savons la couleur des chevaux dans $66 \%$ des cas et leur âge dans $53 \%$ des cas.

18. Greffe d'Antoine Adhémar, 12 novembre 1696, greffe d'Antoine Adhémar, 28 octobre 1697. Les deux contrats dont il est question ont été passés entre l'échec des négociations de paix de 1688-1695 et celles qui conduiront à la Grande Paix de Montréal. Il est donc possible que le souvenir des derniers raids iroquois ait pesé lourd dans la mémoire de notre bailleur. Gilles Havard, "Guerres et paix au XviI ${ }^{\mathrm{e}}$ siècle», La Grande Paix de Montréal de 1701, les voies de la diplomatie franco-amérindienne (Montréal, Recherches amérindiennes au Québec, 1992), 49-59.

19. Greffe Pierre Raimbault, 6 janvier 1705. 
TABLEAU 3

Durée, en années, des contrats de location selon l'animal loué

\begin{tabular}{c|r|r|r|r|r|r|r|r|c}
\hline & \multicolumn{7}{|c|}{ Nombre d'années } & \multirow{2}{*}{} \\
\cline { 2 - 9 } & - de 1 & $\mathbf{1}$ & $\mathbf{1}$ à $\mathbf{2}$ & $\mathbf{2}$ & $\mathbf{3}$ & $\mathbf{4}$ & $\mathbf{5}$ et + & indéterminée & Total \\
\hline Vaches & 2 & 11 & 0 & 8 & 57 & 11 & 8 & 1 & 98 \\
Bœuf* & 10 & 6 & 2 & 2 & 4 & 0 & 1 & 1 & 26 \\
\hline Total & 12 & 17 & 2 & 10 & 61 & 11 & 9 & 2 & 124 \\
\hline
\end{tabular}

* taureaux inclus

\section{PROFIL SOCIOPROFESSIONNEL DES PROPRIÉTAIRES ET DES PRENEURS DE BÉTAIL}

L'identité des vendeurs et des bailleurs, tout comme des acheteurs et des locataires, nous apparaît une donnée fondamentale pour comprendre le rôle que pouvait avoir le bétail comme moyen d'échange. Nous espérons, par l'étude des catégories professionnelles auxquelles appartiennent les propriétaires, mieux connaître ceux qui transigent le bétail. Il sera aussi intéressant de voir s'il existe des distinctions entre le profil des vendeurs et celui des bailleurs. Dans le même sens, il faudra déterminer quelles sont les professions des locataires et si elles rejoignent celles des personnes qui achètent des animaux.

\section{Les propriétaires}

Notre corpus comporte 155 vendeurs ou bailleurs différents. La majorité d'entre eux, plus précisément 124 (80\%), n’apparaissent qu'une seule fois dans nos données, alors que 20 (13\%) signent deux actes et seulement 11 (7\%) individus passent 3 contrats et plus devant les notaires. Les vendeurs et bailleurs exercent des activités diverses, relevant de tous les groupes sociaux ${ }^{20}$. Ils sont habitants, artisans de toutes sortes, bouchers, chirurgiens, notaires, huissiers, marchands, volontaires, seigneurs (tableau 4). Certaines

20. Les professions des individus, comme nous l'avons vu plus haut, sont indiquées dans $50 \%$ des cas. Pour les fins de notre analyse, nous avons tenté de compléter les informations manquantes à l'aide de Parchemin, la banque de données notariales du Québec ancien (1635-1775), ainsi que d'Adhémar, une base de données sur la population et le bâti de Montréal aux xvII et $\mathrm{xVIII}^{\mathrm{e}}$ siècles créée par le Groupe de recherche sur Montréal sous les auspices du Centre canadien d'architecture. Dans certains cas, il a été impossible de déterminer avec exactitude la profession d'individus, par exemple lorsque plusieurs personnes portent le même nom et que nous ne possédons pas d'informations comme le lieu de résidence ou le nom de la conjointe. 
tendances se dessinent toutefois. On remarque que l'élite montréalaise, groupe dont les membres ne représentent en fait qu'une petite partie de la population totale, est très bien représentée. Ainsi, 22 marchands, 6 officiers de justice, 15 bourgeois, nobles et militaires forment $28 \%$ des vendeurs et des bailleurs, $31 \%$ si l'on ne tient pas compte des individus aux professions indéterminées. Les paysans, on s'en doute, représentent le groupe le plus imposant, soit $34 \%$ des propriétaires (38\%, les indéterminés exclus), mais compte tenu de leur poids réel, ils demeurent sous-représentés. Notons cependant qu'ils se démarquent nettement, au sein du groupe des vendeurs, avec $40 \%$ d'entre eux. Il s'agit d'ailleurs du seul groupe professionnel qui vend plus de bêtes qu'il n'en loue. Les artisans, qui occupent des fonctions spécialisées à caractère urbain, vendent ou louent peu de bêtes ( $9 \%$ des vendeurs/bailleurs). Par contre, les bouchers qui entretiennent une relation particulière avec le bétail, leur matériel de travail, forment $5 \%$ des propriétaires. Quatre individus seulement dans nos documents apparaissent comme volontaire, voyageur ou soldat. Ces activités qui supposent une plus grande mobilité, voire instabilité, nuit certainement à l'acquisition de biens, quels qu'ils soient. Notons toutefois qu'il s'agit la plupart du temps d'activités temporaires, les soldats et volontaires devenant souvent par la suite agriculteurs. Enfin un groupe qui nous semble non négligeable est celui des veuves, qui représentent $10 \%$ des vendeurs/bailleurs.

TABLEAU 4

Répartition des vendeurs/bailleurs selon leur profession

\begin{tabular}{l|r|r|r|r|r|r}
\hline \multirow{2}{*}{\multicolumn{1}{c|}{ Profession }} & \multicolumn{2}{|c|}{ Vendeurs } & \multicolumn{2}{c|}{ Bailleurs } & \multicolumn{2}{c}{ Vendeurs/bailleurs } \\
\cline { 2 - 7 } & Nb & $\%$ & Nb & $\%$ & Nb & $\%$ \\
\hline Artisans & 4 & 6 & 11 & 12 & 15 & 9 \\
Bouchers & 3 & 5 & 5 & 5 & 8 & 5 \\
Marchands & 8 & 13 & 14 & 15 & 22 & 14 \\
Nobles et bourgeois & 3 & 5 & 12 & 13 & 15 & 10 \\
Officiers de justice & 2 & 3 & 4 & 4 & 6 & 4 \\
Paysans & 25 & 40 & 28 & 30 & 53 & 34 \\
Veuves & 6 & 10 & 9 & 10 & 15 & 10 \\
Volontaires et & 1 & 2 & 3 & 3 & 4 & 3 \\
voyageurs & 10 & 16 & 7 & 8 & 17 & 11 \\
Indéterminé & 62 & 100 & 93 & 100 & 155 & 100 \\
\hline
\end{tabular}


L'importante participation de l'élite dans la vente et la location de bétail, et dans une moindre mesure des habitants, se présente de façon plus forte encore lorsqu' on observe le groupe des propriétaires ayant contracté deux actes et plus. En effet, sur 20 vendeurs ou bailleurs ayant signé deux contrats, l'on retrouve deux marchands, un officier de justice, cinq bourgeois, nobles ou militaires, deux artisans, un boucher, six paysans et trois veuves. L'élite compose $40 \%$ de ces vendeurs ou bailleurs. Ce même groupe forme $55 \%$ des vendeurs ou bailleurs ayant contracté trois actes ou plus. Le nombre d'habitants et d'artisans ${ }^{21}$ y demeure plutôt constant formant respectivement $30 \%$ et $15 \%$ des vendeurs/bailleurs rencontrés deux fois dans le corpus et $27 \%$ et $18 \%$ des signataires de trois contrats et plus.

Existe-t-il des distinctions entre le profil des vendeurs et celui des bailleurs? Dans les deux cas, le propriétaire de l'animal veut retirer le plus d'avantages possibles de la transaction. Nous avons observé que le choix entre la vente et la location est influencé par l'animal concerné. Dans le cas des vaches, elles sont plus louées que vendues (tableau 1). La raison en est simple. Pour le propriétaire, il est plus rentable de louer une vache. La location épargne le coût d'entretien et procure un revenu en argent ou en livres de beurre en plus de la moitié de la progéniture, et ce, chaque année pour une durée moyenne de trois ans. Vendre une vache, dont la valeur tourne autour de 50 livres est moins avantageux ${ }^{22}$. À l'opposé, le cheval est dans la plupart des cas vendu. Animal beaucoup moins répandu à l'époque de la Nouvelle-France que la vache ou le bouf ${ }^{23}$, le cheval sert plus à charroyer et à se déplacer qu'à labourer ${ }^{24}$ et sa viande n'est consommée qu'en temps de disette. Comme en plus il ne peut pas être loué en échange de lait ou de laine, sa vente en est plus aisée. Le cas des bœufs diffère puisque ceux-ci sont pratiquement autant vendus que loués. Toutefois, lorsque cet animal fait l'objet d'un bail, celui-ci est généralement de courte durée, pour la période des labours par exemple. Il s'agit alors d'une transaction avantageuse pour le locataire qui obtient ainsi la force de traction nécessaire à l'exécution de ses durs travaux agricoles, tout comme pour le bailleur qui profite momentanément d'une augmentation de ses revenus.

21. Les bouchers sont ici inclus dans le groupe des artisans.

22. Selon Jean-Marc Moriceau, op. cit., 23-24, la location de vaches était un bon placement aussi bien pour les bourgeois de la ville, compte tenu de la valeur des bêtes, que pour les hommes de la campagne.

23. Il faut attendre 1710-1715 pour qu'il y ait un cheval en moyenne par exploitation. Louise Dechêne, op. cit., 319.

24. Ibid. 


\section{Les preneurs}

Notre corpus comprend plus d'acheteurs et locataires que de vendeurs ou bailleurs, soit 178. Les preneurs font aussi partie de groupes professionnels variés (tableau 5). Cependant, la répartition de leurs activités diffère de celle des propriétaires. Un nombre considérable d'acheteurs ou de locataires sont agriculteurs, soit dans $56 \%$ des cas (ou $66 \%$ en excluant les gens aux professions indéterminées). Les artisans viennent ensuite avec $12 \%$ des cas (ou 14\%, indéterminés exclus). L'élite représente $11 \%$ des preneurs (14\%, indéterminés exclus).

Comme dans le cas des propriétaires, certains moyens (deux actes) et fréquents (trois actes et plus) acheteurs ou locataires peuvent être identifiés. Le premier groupe est composé de 18 individus, alors que le second en comprend 11. Si les paysans représentent toujours $55 \%$ des moyens et $45 \%$ des fréquents preneurs, nous remarquons une hausse considérable des membres de l'élite dans la catégorie contractant plusieurs actes. Ainsi, les marchands, officiers de justice, nobles et bourgeois représentent $16 \%$ des preneurs moyens et $36 \%$ des fréquents acheteurs / locataires.

Le profil de l'acheteur diffère quelque peu de celui du locataire. En effet, pour pouvoir acheter un animal, il faut posséder un minimum d'argent ou de biens à échanger, condition que ne satisfont pas tous les preneurs. En

TABLEA U 5

Répartition des acheteurs/locataires selon leur profession

\begin{tabular}{l|r|r|r|r|r|r}
\hline \multirow{2}{*}{\multicolumn{1}{c|}{ Profession }} & \multicolumn{2}{|c|}{ Acheteurs } & \multicolumn{2}{c|}{ Locataires } & \multicolumn{1}{c}{ Acheteurs/locataires } \\
\cline { 2 - 7 } & Nb & $\%$ & Nb & $\%$ & Nb & $\%$ \\
\hline Artisans & 10 & 16 & 12 & 10 & 22 & 12 \\
Bouchers & 2 & 3 & 0 & 0 & 2 & 1 \\
Marchands & 6 & 10 & 4 & 3 & 10 & 6 \\
Nobles et bourgeois & 3 & 5 & 2 & 2 & 5 & 3 \\
Officiers de justice & 2 & 3 & 2 & 2 & 4 & 2 \\
Paysans & 23 & 38 & 77 & 66 & 100 & 56 \\
Veuves & 3 & 5 & 3 & 2 & 6 & 4 \\
Volontaires et & 1 & 2 & 1 & 1 & 2 & 1 \\
voyageurs & 11 & 18 & 16 & 14 & 27 & 15 \\
Indéterminé & 61 & 100 & 117 & 100 & 178 & 100 \\
\hline
\end{tabular}


effet, selon leur groupe professionnel, ils tendent à adopter l'une ou l'autre des solutions pour prendre possession du bétail de manière durable ou temporaire. Dans la majorité des cas, les paysans louent les animaux (dans $66 \%$ des cas), alors que les quelques membres de l'élite les achètent. Par exemple, sur les dix marchands échantillonnés, trois louent des bêtes, six en achètent et un seul signe ces deux types d'actes. Des nuances doivent cependant être parfois apportées à l'intérieur de ces groupes selon les métiers exercés par les individus. Le groupe des artisans, par exemple, achète pratiquement autant d'animaux qu'il en loue. Et si l'on isole les contrats passés par les bouchers et ceux signés par les charretiers, on voit que les premiers achètent des bœufs, tandis que les seconds acquièrent des chevaux. De telles distinctions ont bien entendu des motivations essentiellement professionnelles.

Bref, nous avons observé d'une part que les propriétaires qui transigent des animaux forment un groupe socialement très diversifié. Chacun d'eux investit dans le bétail à des degrés différents et utilise la vente et la location des bêtes de manière à faire le plus de bénéfices possible, peu importe les raisons qui les motivent. L'élite montréalaise demeure toutefois un groupe des plus actifs dans ces types de transactions. D'autre part, le groupe des preneurs est constitué d'un plus grand nombre d'individus aux occupations beaucoup moins diversifiées, la majorité d'entre eux étant des paysans. Une distinction importante se dessine entre les locataires, plus souvent qu'autrement des agriculteurs n'ayant pas les moyens financiers d'acheter les bêtes, et les acheteurs, souvent membres de l'élite qui détiennent les capitaux nécessaires à leur acquisition. Disons enfin que ce découpage, essentiel pour la suite de notre analyse, mériterait d'être raffiné pour déterminer plus précisément les conditions socio-économiques de tous les individus de notre corpus afin d'établir des distinctions certaines entre les membres d'un même groupe professionnel.

\section{DIVERSITÉ DES SITUATIONS}

Suivant la diversité sociale des vendeurs et des bailleurs ainsi que des acheteurs et des locataires, il n'existe pas qu'un seul modèle de transaction impliquant un riche propriétaire et un preneur misérable, mais plutôt une diversité de situations. À l'aide d'exemples, nous tenterons maintenant de tracer un éventail des situations possibles.

Dans la majorité des cas, les contrats de vente et de location sont passés entre des paysans ou des artisans n'apparaissant qu'une seule fois dans ce type de marché et qui se servent de ce moyen d'échange pour combler 
un besoin ou pour répondre à une obligation. Dans certains cas, le propriétaire veut ainsi rembourser une dette à un voisin ou à un membre de la famille. C'est le cas de Jean Laroche, un agriculteur, qui vend un cheval à son frère Gabriel pour 100 livres. Ce montant servira à payer une partie de la part de terre achetée par Jean à Gabriel pour un prix de 150 livres ${ }^{25}$. Dans le même sens, Jean César dit Lagardette, de Boucherville, qui doit 30 livres à son gendre Jacques Viger, charpentier de bateaux pour le roi, lui vend une vache de même valeur et leurs comptes sont quittes ${ }^{26}$. D'autres veulent se procurer, en échange de l'utilisation de leur animal, des denrées spécifiques. Par exemple, Jean Cailloud dit Baron vend un cheval à Jean De Lahaye en échange de 230 livres de tabac ${ }^{27}$. Un animal peut aussi être vendu ou loué par son propriétaire en échange d'un service. Ainsi, Nicolas Chapput, qui a loué deux bœufs de Pierre Chesne pour deux ans, s'engage à faire les labours de ce dernier pendant ces deux années ${ }^{28}$. L'hivernage de bêtes peut devenir une condition de vente ou de location. Le 4 décembre 1721, Jean Coitoux dit St-Jean vend un cheval à son fils Pierre qui doit en retour hiverner une vache pour trois hivers ${ }^{29}$. Il faut comprendre que cette saison est la période la plus difficile pour la survie des animaux qui risquent de mourir de froid ou d'un manque de nourriture. En plus, en cette période de l'année, la majorité des animaux sont moins productifs et demandent plus de soins. Lorsque l'habitant pouvait s'éviter d'hiverner quelques-unes de ces bêtes, il s'agissait d'une bonne affaire. Il arrive enfin, tout simplement, qu'un individu, pour éviter de débourser les coûts d'entretien d'un animal, le loue ou encore le vende, lorsque son cheptel croît suffisamment pour combler les besoins que nécessite son exploitation agricole.

Il arrive aussi qu'un paysan, se retrouvant momentanément dans une situation économique précaire, multiplie les transactions d'animaux pour subvenir à ses besoins et à ceux de sa famille. C'est le cas de Mathurin Langevin dit Lacroix. Ce dernier est un paysan qui contracte quatre contrats de vente ou de location en 1690. Quelque temps auparavant, Mathurin Langevin a perdu son épouse. La vie paysanne, exigeante, demande le partage des tâches entre l'homme et la femme. Il devient difficile pour un seul membre du couple d'accomplir les nombreuses tâches

25. Greffe de Michel Lepailleur dit Laferté, le 30 septembre 1715.

26. Greffe d'Antoine Adhémar, 17 juin 1704.

27. Greffe d'Antoine Adhémar, 14 juin 1705.

28. Greffe de Claude Maugue, le 4 février 1692.

29.Greffe de Nicolas Senet dit Laliberté le 4 décembre 1721. 
de la vie quotidienne ${ }^{30}$. Langevin loue et vend par conséquent une partie de son bétail ${ }^{31}$ pour obtenir en échange de l'argent, du blé ou l'entretien d'un de ses enfants. Ainsi, le 11 avril, une ordonnance du bailli décrète la vente d'un poulain, appartenant à Mathurin Langevin, à Charles Gervaise qui doit fournir au vendeur six minots de blé, mais qu'il conservera jusqu'aux semences prochaines. Le 28 octobre, Langevin vend une pouliche à André Huneault. Ce dernier paie son acquisition 30 livres en plus de prendre, pour les 20 livres restant à débourser, un enfant Langevin en pension pour 2 mois. Une clause du contrat indique que dans un mois, la mère de la pouliche sera louée à son tour pour 6 mois pour 40 livres que le preneur remboursera en entretenant le fils déjà pensionné. Le 6 décembre, le même Langevin loue à Pierre Huneault un cheval pour six mois. Il demande en contrepartie au preneur de nourrir, soigner et laver son fils de 6 ans, Antoine Langevin, et de pouvoir employer le cheval pour une journée. Trois jours plus tard, c'est Louis Hurtubise qui s'engage à nourrir en blé froment Charles Langevin, le fils de 3 ou 4 ans du bailleur, en échange de l'utilisation pour 6 mois d'une cavale. Mathurin Langevin pourra employer la bête deux jours pendant la durée de la location ${ }^{32}$.

Dans le même ordre d'idées, les transactions concernant plusieurs bestiaux à la fois sont souvent le fruit de deux situations particulières. Il n'est pas rare qu'une femme devenant veuve vende ses bêtes à un marchand ou à des membres de la famille ${ }^{33}$. À titre d'exemple, le 4 janvier 1700, Marie Chauvin, veuve de Nicolas Baron, vend deux taureaux de 3 ans, un bœuf et une vache pour 255 livres à Charles de Couagne, marchand de Montréal. Ce dernier loue immédiatement la vache pour 3 ans à Marie Chauvin contre 10 livres de beurre par an et le partage de la progéniture à venir ${ }^{34}$. Les règlements de succession impliquent aussi parfois la vente des bestiaux. Une autre raison de vendre ou louer ses animaux demeure encore une fois l'endettement. Ainsi, René Goulet vend plusieurs de ses bêtes à Charles Juchereau pour rembourser une dette de 635 livres contractée envers l'acheteur. Cependant

30. Voir, entre autres, Jan Noel «New France : les femmes favorisées», Atlantis, 7 (1981) : 82-83. 31. Au recensement de 1681, Mathurin Langevin possède six bêtes à corne. Comme il vend surtout des chevaux, il est possible de croire qu'il possédait malgré ses ventes et locations toujours un bon nombre de bêtes à corne.

32. Greffe de Claude Maugue, les 28 octobre 1690 et 6 décembre 1690, greffe d'Antoine Adhémar les 11 avril 1690 et 9 décembre 1690 .

33. Dans notre corpus cinq veuves vendent leurs bestiaux soit à Charles de Couagne, marchand, soit à Charles Juchereau, écuyer, soit à leur famille.

34. Greffe d'Antoine Adhémar, le 4 janvier 1700. 
le meilleur exemple demeure celui de Pierre Jannot dit Belhumeur. Le $1^{\mathrm{er}}$ mars 1704, Jannot vend ses six bœufs et trois vaches à Charles de Couagne pour 380 livres. Le marchand paie le montant dû en acquittant les dettes de Jannot à Paul Bouchard et à François Beauchamp, en plus de rembourser une partie des sommes que lui doit le vendeur. Ce contrat est suivi d'un acte de location des mêmes bestiaux par Charles de Couagne à Pierre Jannot pour une valeur de 234 livres sur 3 ans. Une clause indique que Jannot, à la fin des trois années, pourra racheter ses bêtes à de Couagne en lui remettant 380 livres. La condition économique de Jannot ne semble pas s'être améliorée durant ces trois années, puisque le 8 mars 1708, ce dernier vend quatre bœufs, une vache, une taure et un poulain, cette fois à Paul Bouchard. Le notaire mentionne que le vendeur, qui devait encore de l'argent à Charles de Couagne lors du décès de ce dernier en 1706, a dû, pour s'acquitter de sa dette à la succession, vendre ses bestiaux au sieur de Repentigny. Mais comme il ne pouvait faire valoir sa terre sans bestiaux, il demanda l'aide de Paul Bouchard, qui consentit à lui prêter 200 livres pour l'achat de bêtes. Dans le contrat de 1708, Jannot vend à Paul Bouchard les animaux achetés avec l'argent de ce dernier en retour de leur utilisation gratuite jusqu’à la Saint-Michel, puis encore pour un an à loyer. À la fin du contrat, Jannot payera à Bouchard les 200 livres et 6 livres de loyer par animal sauf pour le cheval pour lequel il ne devra rien. La situation de Jannot n'avait donc rien d'enviable, mais elle prouve néanmoins qu'il existait toujours une façon de s'arranger avec ses créanciers, ceux-ci voulant avant tout récupérer les montants dus ${ }^{35}$.

Dans ces derniers exemples, un individu ressort particulièrement, le marchand. Pour lui, le bétail est un moyen d'échange avantageux, lorsqu'il s'agit d'éponger les dettes de sa clientèle et devient ensuite par la location un placement rentable ${ }^{36}$. Les membres de l'élite, groupe dont font partie ces marchands, forment la majorité des moyens et fréquents bailleurs ainsi que des acheteurs et sont presque toujours présents dans les ventes et les baux de plusieurs animaux. Nous n'avons qu'à penser à Charles de Couagne, un des marchands les plus importants à la fin du XviI siècle à Montréal, et intervenant dans 11 contrats, ou à Jean Petit dit Boismorel, huissier et bourgeois, qui a signé 15 actes. Ces membres de

35. Greffe de Michel Lepailleur dit Laferté, le $1^{\text {er }}$ mars 1704 et le 8 mars 1708.

36. Louise Dechêne, op. cit., 318, souligne que la saisie des bestiaux étant interdite dans la colonie à partir de 1662, il était fréquent que les marchands contournent l'interdiction en achetant puis louant les bêtes au vendeur endetté. 
l'élite possèdent certainement plusieurs des plus gros troupeaux de Montréal; c'est le cas de Jacques Leber.

\section{CONCLUSION}

Nous avons vu que le bétail s'inscrit dans les relations économiques des résidants de l'île de Montréal, peu importe les professions que ceux-ci exercent. Le bétail acquiert toutefois davantage d'importance comme moyen d'échange pour les paysans ainsi que pour les membres de l'élite. Nous avons vu que les motivations qui sous-tendent les ventes et les locations de bétail varient grandement selon les individus. Pour les paysans et pour les artisans, il s'agit majoritairement d'un instrument pour se procurer des denrées spécifiques, un service ou pour rembourser une dette à un membre de la famille ou à un voisin. Pour les membres de l'élite, le bétail représente souvent le seul moyen de récupérer des sommes importantes avancées à des individus moins bien nantis, généralement des habitants, et il devient un investissement rentable. Dans ces cas, les transactions de bétail représentent une certaine interface entre la ville, et spécialement ses élites, et la campagne environnante.

Bien entendu, cette analyse n'est qu'une première étape à une étude beaucoup plus complexe sur la possession du bétail en Nouvelle-France. Plusieurs avenues demeurent inexplorées, des sources telles que les recensements, les contrats de mariage, les inventaires après décès, d'autres transactions concernant le bétail, telles que les baux à ferme, pourront donner des précisions intéressantes et conduire à une meilleure compréhension des mécanismes qui sous-tendent le fonctionnement de l'élevage en Nouvelle-France. 